\title{
Multimodal label-free ex vivo imaging using a dual-wavelength microscope with axial chromatic aberration compensation
}

\author{
Andrea Filippi \\ Eleonora Dal Sasso \\ Laura Iop \\ Andrea Armani \\ Michele Gintoli \\ Marco Sandri \\ Gino Gerosa \\ Filippo Romanato \\ Giulia Borile
}




\title{
Multimodal label-free ex vivo imaging using a dual-wavelength microscope with axial chromatic aberration compensation
}

\author{
Andrea Filippi, a,b,c,d,* Eleonora Dal Sasso, ${ }^{\mathrm{e}, \mathrm{f}}$ Laura lop, ${ }^{\mathrm{e}, \mathrm{f}}$ Andrea Armani,, ${ }^{\mathrm{f}, \mathrm{g}}$ Michele Gintoli,, ${ }^{\mathrm{a}, \mathrm{d}}$ Marco Sandri, ${ }^{\mathrm{f}, \mathrm{g}}$ \\ Gino Gerosa, ${ }^{e, f}$ Filippo Romanato, ${ }^{\mathrm{a}, \mathrm{c}, \mathrm{d}, \mathrm{h}, \dagger}$ and Giulia Borile ${ }^{\mathrm{a}, \mathrm{c}, \mathrm{g}, \dagger}$ \\ aUniversity of Padua, Department of Physics and Astronomy "G. Galilei," Padua, Italy \\ ${ }^{\mathrm{b}}$ Fondazione Bruno Kessler, Trento, Italy \\ Institute of Pediatric Research Città della Speranza, Padua, Italy \\ 'Laboratory for Nanofabrication of Nanodevices, Padua, Italy \\ eUniversity of Padua, Department of Cardiac, Thoracic and Vascular Sciences, Padua, Italy \\ 'Venetian Institute of Molecular Medicine, Padua, Italy \\ gUniversity of Padua, Department of Biomedical Sciences, Padua, Italy \\ ${ }^{\mathrm{h}}$ CNR-INFM TASC IOM National Laboratory, Trieste, Italy
}

\begin{abstract}
Label-free microscopy is a very powerful technique that can be applied to study samples with no need for exogenous fluorescent probes, keeping the main benefits of multiphoton microscopy, such as longer penetration depths and intrinsic optical sectioning while enabling serial multitechniques examinations on the same specimen. Among the many label-free microscopy methods, harmonic generation $(\mathrm{HG})$ is one of the most intriguing methods due to its generally low photo-toxicity and relative ease of implementation. Today, $\mathrm{HG}$ and common two-photon microscopy (TPM) are well-established techniques, and are routinely used in several research fields. However, they require a significant amount of fine-tuning to be fully exploited, making them quite difficult to perform in parallel. Here, we present our designed multimodal microscope, capable of performing simultaneously TPM and HG without any kind of compromise thanks to two, separate, individually optimized laser sources with axial chromatic aberration compensation. We also apply our setup to the examination of a plethora of ex vivo samples to prove its capabilities and the significant advantages of a multimodal approach. (C) 2018 Society of Photo-Optical Instrumentation Engineers (SPIE) [DOI: 10.1117/1.JBO.23.9.091403]
\end{abstract}

Keywords: label-free; microscopy; multiphoton; dual-wavelength; chromatic aberration; ex vivo.

Paper 170769SSR received Nov. 25, 2017; accepted for publication Feb. 12, 2018; published online Mar. 7, 2018.

\section{Introduction}

Multiphoton microscopy (MPM) is a laser-scanning microscopy technique based on a nonlinear, strongly localized light-matter interaction, which depends on a higher power of the illumination intensity. MPM does not have its optical sectioning capabilities severely limited by the fluorescence contributions from outside the depth of focus of the objective (as in WideField Microscopy) and, therefore, represents one of the best noninvasive techniques to achieve imaging in deep explanted tissues or in living animals. ${ }^{1,2}$ The most common MPM variation is the two-photon microscopy (TPM), which takes advantage of the quasisimultaneous absorption of two photons by a molecular receptor, in a single quantum event. This phenomenon was theoretically predicted by Maria Goeppert-Mayer in $1931^{3}$ but laid dormant until Denk et al. $^{2}$ devised a practicable two-photon laserscanning fluorescence microscope. Despite the need for very intense light sources, TPM presents several advantages over the classical single-photon techniques. For example, the wavelengths used in TPM, which usually range from near-IR to midIR, are significantly less prone to scattering and absorption from thick specimens (with respect to visible light) and, therefore, exhibit longer penetration depths. Furthermore, due to the

*Address all correspondence to: Andrea Filippi, E-mail: andrea.filippi.5@phd. unipd.it

${ }^{\dagger}$ Contributed equally small two-photon excitation (TPE) focal volume generated by the high photon fluxes required to achieve two-photon absorption (TPA), ${ }^{1}$ TPM has an inherent capability of performing axial sectioning and confines the photo-bleaching effect inside a very limited volume. ${ }^{4}$ Despite the obvious benefits of TPM, there are cases where it cannot (or should not) be applied. When specimens are very susceptible to photo-bleaching and photodamages, even with NIR excitation wavelengths, or when any staining process must be avoided to preserve the specimens in their original conditions (e.g., in case of multitechniques examinations), the use of another nonlinear light/matter interaction-based technique, known as harmonic generation (HG), is preferable. HG retains the intrinsic optical sectioning capabilities of TPM but does not involve the absorption of the excitation light, producing very little heat and resulting in a much less phototoxic approach. Furthermore, HG does not require the use of molecular probes to generate and detect signals from a specific biological feature: any structure that satisfies the prerequisites for at least one order of $\mathrm{HG}$ can produce a detectable $\mathrm{HG}$ signal, thus drastically reducing the specimen preparation requirements and induced alterations. The most commonly used orders of $\mathrm{HG}$ are the second harmonic generation (SHG) and third harmonic generation (THG), which, respectively, doubles and triples the incident light frequency and produce a 
narrow band at half and one-third of the excitation wavelength. Both SHG and THG are very sensitive to external factors (e.g., temperature, medium ionic strength, and $\mathrm{pH}$ ) that can alter, even slightly, the molecular structures from which the signal originates. ${ }^{5}$ Therefore, a careful optimization of the experimental parameters must be performed on a sample basis to achieve the best possible HG experimental conditions. Here, we report on the design and realization of a multimodal microscope capable of performing both TPM and HG simultaneously thanks to two separate laser sources that can be independently optimized for one technique or the other. We also report on the use of our microscope for the study of several thin and thick ex vivo samples, to show the quality and quantity of complementary information that can be extracted through a multimodal approach.

\section{Methods}

All experimental procedures were performed according to the European Commission guidelines and have been approved by the local ethical committee and the Italian authority (Ministero della Salute), in compliance of Italian Animal Welfare Law (Law n. 116/1992 and subsequent modifications).

\subsection{Heart Cryosections}

Mice were sacrificed by cervical dislocation and hearts were quickly harvested and cut in two portions in the transverse direction and processed as described elsewhere. ${ }^{6}$ Blood clots were carefully removed and the heart was fixed to maintain structural integrity with $1 \%$ paraformaldehyde in phosphate buffered saline (PBS 1X: $137 \mathrm{NaCl}, 2.7 \mathrm{KCl}, 10 \mathrm{Na}_{2} \mathrm{HPO}_{4}, 1.8 \mathrm{KH}_{2} \mathrm{PO}_{4}$, in $\mathrm{mM}$ ) at room temperature for $15 \mathrm{~min}$. After three washes for 5 min with PBS $1 \mathrm{X}$, hearts were allowed to dehydrate in sucrose $30 \%$ (w/v in distilled water) at $4^{\circ} \mathrm{C}$ overnight. The following day, hearts were embedded in OCT freezing medium (Optimal Cutting Temperature, Kaltec) and carefully frozen in liquid nitrogen vapor. Frozen samples were maintained at $-80^{\circ} \mathrm{C}$. Frozen hearts were cut in $10-\mu \mathrm{m}$ slices using a cryostat (Leica CM1850, Leica Microsystems GmbH, Wetzlar, Germany) and placed on superfrost glass slides (Vetrotecnica) maintained at $-80^{\circ} \mathrm{C}$ until use.

\subsection{Gastrocnemius Muscle and Succinate Dehydrogenase Staining}

Gastrocnemius muscles were harvested from mice and immediately frozen in liquid nitrogen, maintained at $-80^{\circ} \mathrm{C}$ and used within few days, to minimize NADH fluctuations. Ten-micron cryosections were cut with a cryostat (Leica CM1850, Leica Microsystems $\mathrm{GmbH}$, Wetzlar, Germany), maintaining the same slicing angle for all the sections. Some slices were stained with succinate dehydrogenase (SDH) activity, whereas others were directly observed in 2PM. SDH cryosections were also examined under a fluorescence microscope (Olympus BX60), as described elsewhere. ${ }^{7}$

\subsection{Ex Vivo Lungs}

Mice were sacrificed by cervical dislocation and lungs were quickly harvested and carefully washed with ice-cold PBS to avoid blood clotting. Lungs were then transferred to a Petri dish filled with PBS. To prevent curling and movement, lungs were held down by a homemade platinum holder.

\subsection{Decellularized Bovine Pericardium}

Bovine pericardia were collected from the local slaughterhouse and decellularized using a method based on alternated hypo- and hypertonic solutions, detergents (Triton X-100 and sodium cholate, Sigma-Aldrich, Saint Louis, Missouri) and nonspecific endonucleases (Benzonase, Sigma-Aldrich). ${ }^{8,9}$ Following the decellularization procedure, samples of $1 \mathrm{~cm}^{2}$ were placed into plastic embedding devices (Bio Optica, Milano, Italy) and covered by a thin layer of $4 \%$ low melting agarose solution prepared in PBS (Sigma-Aldrich).

\subsection{Statistical Analysis}

Images were processed in Fiji using the ROI Manager plug-in to obtain mean ROI intensities and area values for every single hand-drawn region of interest, delimiting the exposed fibers cross sections. Pearson correlation coefficient was evaluated in OriginPro ${ }^{\mathrm{TM}} 2016$ and data were fitted by linear regression.

\subsection{Data Availability}

The datasets generated during and/or analyzed during the current study are available from the corresponding author on reasonable request.

\section{Results}

\subsection{Microscopy Setup Design and Optimization}

The main laser source is a mode-locked Ti:Sapphire pulsed laser (Chameleon Ultra II, Coherent Inc.), with pulses of $\sim 140$ fs at $80 \mathrm{MHz}$ and tunable emission wavelength of 700 to $900 \mathrm{~nm}$, which also serves as a pump for the second laser source, an Optical Parametric Oscillator (Compact OPO, APE) with tunable emission wavelength in the 1000- to 1550 -nm range. Both lasers pass through a pair of Pockels Cells, voltage-controlled wave plates that combined with a polarizer can modulate the laser beam power with up to $100 \mathrm{kHz}$ frequency (360-80 and 350-80, ConOptics Inc.). Two mechanical shutters are used as hard beam-blocking devices (SH05/M, Thorlabs Inc.). A couple of silver-coated mirrors (PF10-03-P01, Thorlabs Inc.) deflect them to the recombination area, where they are spatially overlapped by means of another silver mirror (PF10-03-P01, Thorlabs Inc.) and a dichroic mirror (DMSP1000, Thorlabs Inc.) mounted on piezo-controlled kinematic mirror mounts (POLARIS-K1PZ, Thorlabs Inc.). Two telescopes (\#59-134, Edmund Optics) are used for the axial chromatic aberration compensation. Both telescopes have a $3 \times$ magnification to increase the diameter of the beams from 2.5 to $7.5 \mathrm{~mm}$. The larger diameter, in combination with another $3 \times$ magnification from the scanning unit optics, allows for a slight overfill of the objective back-aperture $(22 \mathrm{~mm})$. From the recombination area, the beams enter the scanning head (Bergamo Series, Thorlabs Inc.), equipped with a scan lens/tube lens couple (SL50-2P2 and TL200-2P2, AR coating and color correction range 680 to $1600 \mathrm{~nm})$ and a Galvo/Resonant scanner $(8-\mathrm{kHz}$ scanning rate) and arrive on the sample through a water-immersion objective (XLPLN25XWMP2, Olympus, NA 1.05, water immersion, color corrected up to $1300 \mathrm{~nm}$ ). The polarization state of both laser beams can be controlled independently by two achromatic $\lambda / 4$ wave plates preceded by two achromatic $\lambda / 2$ wave plates (AHWP05M-600/-980 and AQWP05M-600/-980, Thorlabs Inc.), which can compensate the polarization variations induced 
by the optical components in the scanning head to achieve the desired polarization state before the objective back-aperture with an high grade of purity. The light scattered or emitted from the sample is collected in epi-direction and, thanks to a longpass dichroic mirror (705-nm cutoff, Semrock), is diverted to the detection module. This module consists of $4 \times$ GaAsP PMT detectors (H7422-40, Hamamatsu), with bandpass filters (395/25-nm Chroma, 460/50-nm Chroma, 525/40-nm Semrock, and 625/90-nm Semrock) spectrally separated by $3 \times$ dichroic mirrors (425-nm Chroma, 495-nm Chroma, and 565-nm Chroma). The transmitted light can be collected by a condenser lens (D-CUO Achr-Apl, 1.4 NA, Nikon) and detected by a biased InGaAs detector (DET20C, Thorlabs Inc.) positioned below the sample holder. We keep a constant laser power across all experiments of $10 \mathrm{~mW}$ for the pump and $15 \mathrm{~mW}$ for the OPO (measured on the objective back-aperture). A schematic representation of our setup can be seen in Fig. 1 .

To optimize the polarization state for both laser beams and achieve the best possible quality of image, we positioned a polarization analyzer (SK010PA-NIR, Schäfter+Kirchoff) under the scanning-head of the microscope to measure the polarization before the back-aperture of the objective and we rotated a couple of $\lambda / 2$ wave plate and $\lambda / 4$ wave plate (AHWP05M-600/-980 and AQWP05M-600/-980, Thorlabs Inc.), setting the polarization of both lasers to circular. A circular polarization ensures the highest signal for the autofluorescence emission due to the generally random orientation of the intrinsic fluorophores dipole axis ${ }^{10}$ while also maximizing the SHG signal for randomly oriented collagen fibers. If the collagen structures of some samples happen to have a preferred orientation, the polarization state can be easily switched to a linear polarization and aligned to be at $45 \mathrm{deg}$ from the fibers, which is the orientation of maximum SHG signal generation. ${ }^{11,12}$ The polarization state was then checked using a commercial calibration sample (F24630,
Thermofisher), following the procedure described by Chen et al. ${ }^{13}$ The depolarizing effect of the objective proved to be not significantly affecting the circular polarization state on the sample plane, at least for our purposes. In case of highprecision measurements, specific interest over slight variations of the polarization state or 3-D polarization imaging, a much more precise control would be needed, requiring complete mapping of in-and-out polarization. ${ }^{14,15} \mathrm{~A}$ known major issue of dual-wavelength excitation is chromatic aberration. To fully compensate for the axial chromatic aberration, we chose an objective with a reported color correction up to $1300 \mathrm{~nm}$ (XLPLN25XWMP2, Olympus), as well as a pair of scan lens/tube lens (SL50-2P2 and TL200-2P2) color corrected in the 680 - to 1600 -nm range, and we adopted a procedure commonly used in STED Microscopy, where perfect overlapping of two beams focal volumes is a strictly required condition. ${ }^{16-18}$ Following the STED procedure, we fine-tuned the axial aberration via a couple of telescopes (\#59-134, Edmund Optics) positioned right before the wave plates in the beams' optical paths, which allowed us to shift the two focal volumes along the $z$-axis by slightly changing the curvature radii of the two beams, thus obtaining a high-precision superposition. We also corrected the lateral focal volumes displacement by carefully superimposing the two point spread functions (PSFs) via a silver mirror (PF10-03-P01, Thorlabs Inc.) and a dichroic mirror (DMSP1000, Thorlabs Inc.) mounted on piezo-controlled kinematic mirror mounts (POLARIS-K1PZ, Thorlabs Inc.). We measured the two PSFs by imaging a sample of PbS Quantum Dots (average diameter $70 \mathrm{~nm}$, up to 1300 -nm 2p-Absorption, $\sim 650$-nm Emission) dispersed in a sol-gel $\mathrm{ZrO}_{2}$ matrix. We checked the status of the axial chromatic aberration and the lateral displacement iteratively through the analysis of several $z$-stack of the PSFs using a custom-developed software (Fig. 2).

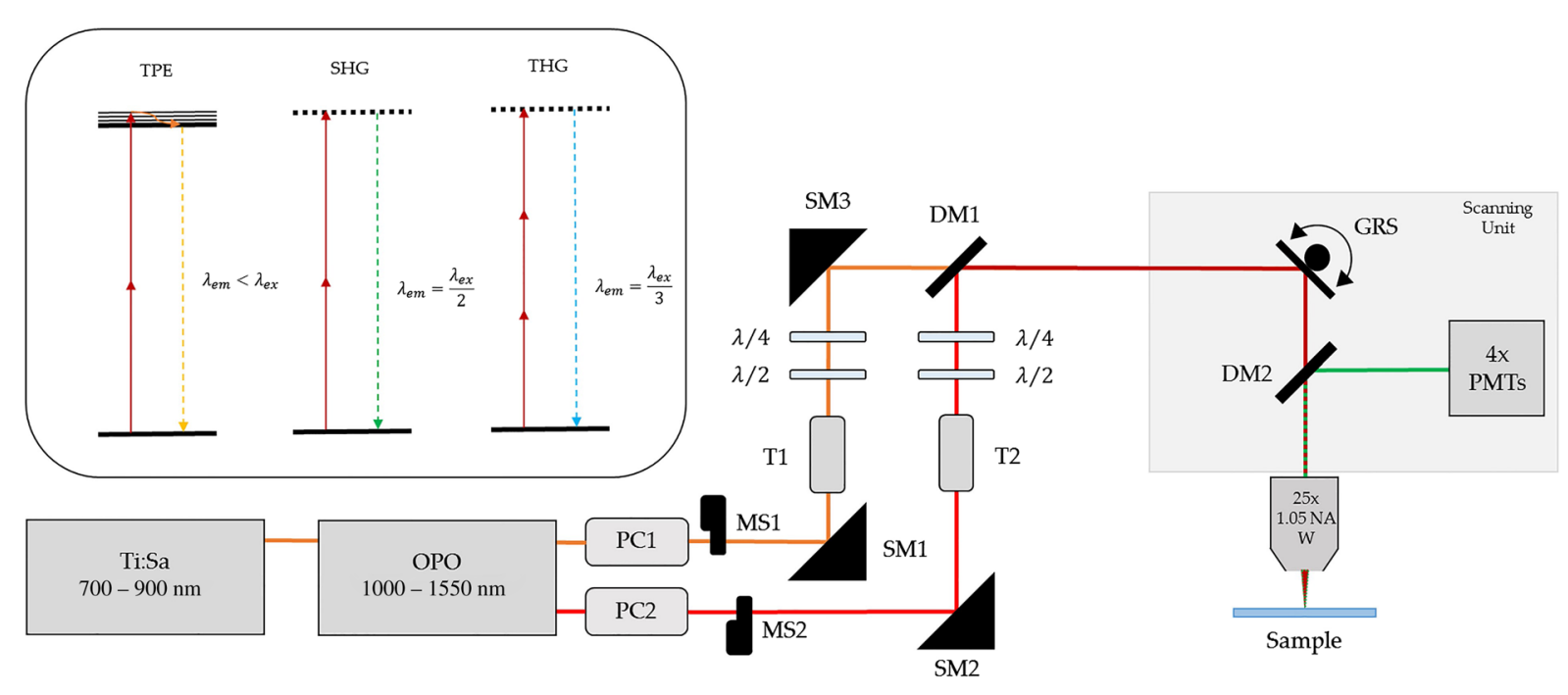

Fig. 1 Schematic representation of the setup. Both laser beams coming from the Ti:Sa laser and the $\mathrm{OPO}$ are modulated in intensity by two Pockels cells (PC1, PC2), circularly polarized by a couple of half-wave plates and quarter-wave plates $(\lambda / 2, \lambda / 4)$, spatially overlapped by means of a silver mirror (SM3) and a dichroic mirror (DM1) mounted on piezo-controlled kinematic mirror mounts, and finally focused on the sample through a 25x Objective. The fluorescence and HHG signals are detected in epidirection and sent to four GaAsP PMT detectors thanks to another dichroic mirror inside the scanning unit (DM2). Two telescopes (T1, T2) are used for the axial chromatic aberration compensation. Two mechanical shutters are used as hard beam-blocking devices (SH05/M, Thorlabs Inc.). Two silver mirrors (SM1, SM2) deflect the beams to the recombination area. 

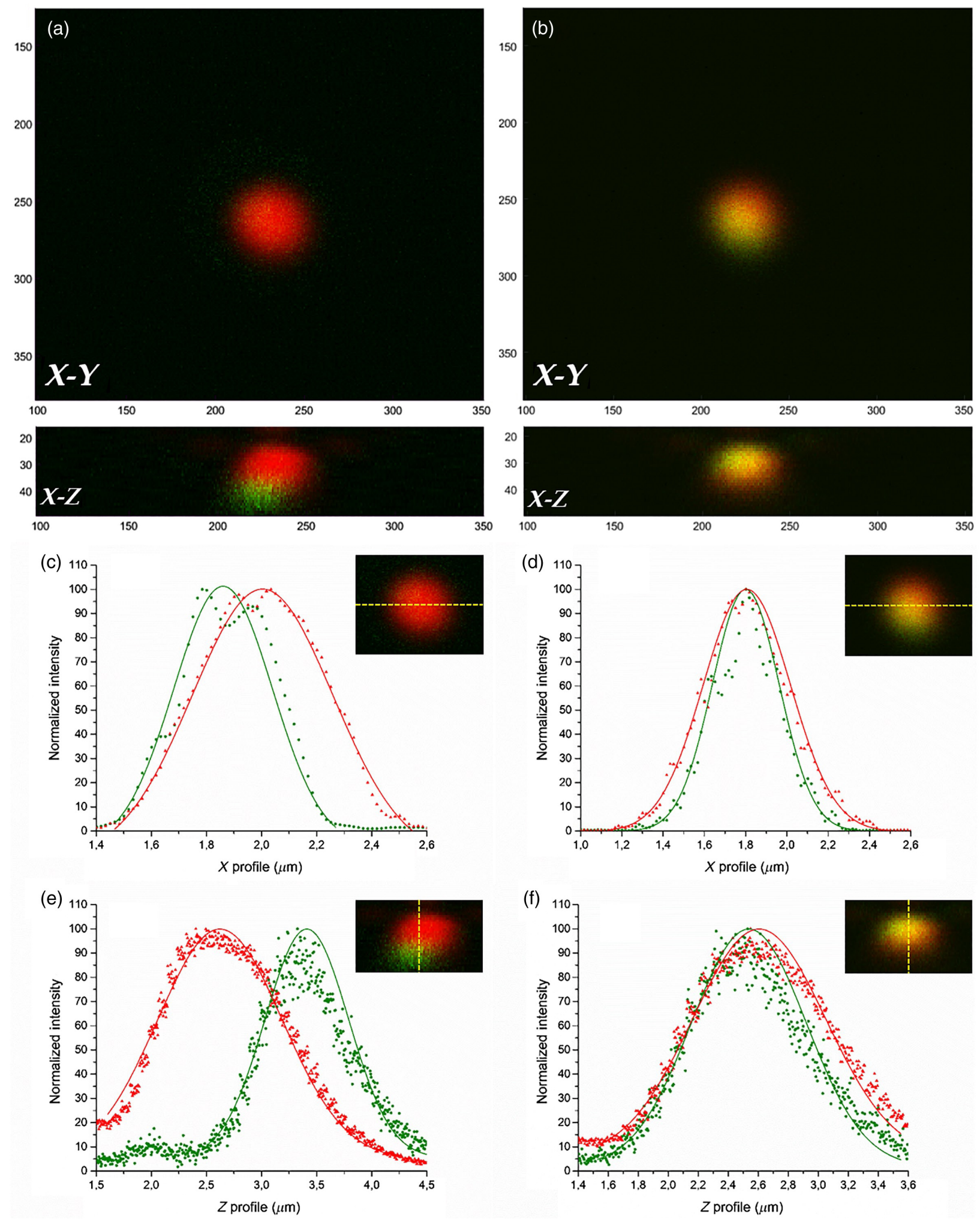

Fig. 2 PSFs displacement comparison before and after correction. (a) PSFs displacement in the $X-Y$ (top) and $X-Z$ (bottom) planes before fine-tuning. (b) PSFs displacement in the $X-Y$ (top) and $X-Z$ (bottom) planes after fine-tuning. (c) and (e) show the $X$ and $Z$ profiles of the two PSFs before fine-tuning, while (d) and (f) show their profiles after fine-tuning (the insets show qualitatively the direction of the profiles). The final lateral displacement is $(5 \pm 7) \mathrm{nm}$, thus negligible, while the residual axial displacement is $(112 \pm 48) \mathrm{nm}$, well below the minimum requirements for simple MPM. Axis scales express coordinates in pixels $(4.17 \mathrm{~nm} /$ pixel $X$ and $Y, 100 \mathrm{~nm} /$ pixel $Z)$. 
The software controlled two mechanical shutters (SH05/M, Thorlabs Inc.) via an Arduino microcontroller board (Arduino MEGA, Arduino) connected to the shutters controllers (KSC101, Thorlabs Inc.). The open/close cycles of the shutters were synchronized with the $z$-stack acquisition process to perform simultaneously two $z$-stacks at two different wavelengths, minimizing any possible mechanical drift contribution to the determination of the PSFs relative position, achieving a very high precision. The synchronization was done by registering 60 separate frames for every $z$-plane of a $z$-stack that extended about $2 \mu \mathrm{m}$ below and above the approximate position of the two PSFs ( $z$-plane separation of $0.4 \mu \mathrm{m}$ ) and timing the aperture/closure of the mechanical shutters to have 20 frames of background signal (both shutters closed), 20 frames of a $\mathrm{PbS}$ QD illuminated by one beam, and 20 frames of the same $\mathrm{PbS}$ QD illuminated by the other beam. Afterward, we could import the frames of a $z$-stack inside the software, selecting properly the ranges associated with the background and the two beams, and obtain a highly precise 3-D representation of the relative position of the two PSFs. After careful fine-tuning, we achieved a full 3-D PSFs superimposition, with a negligible residual lateral displacement and an axial displacement of $<200 \mathrm{~nm}$, near the minimum requirement for STED applications. ${ }^{19}$ Through the previously described procedure, we could fully compensate for the axial chromatic aberration; however, a recent study $^{20}$ showed that there is a nonnegligible contribution to image degradation also from the transverse chromatic aberration, which varies within the field of view, up to $\approx 1.5 \mu \mathrm{m}$ at the edge of the field of view (FOV $484 \times 484 \mu \mathrm{m}$ ). We did not compensate for this type of chromatic aberration; however, we are planning to do so in our future works.

\subsection{Label-Free Analysis of Thin Cryosections}

To test the quality of the obtainable images after the polarization calibration, we imaged mice heart cryosections at 800 and $1200 \mathrm{~nm}$, using both laser beams (Fig. 3).

The cryosections showed overall high signal intensity and very good $\mathrm{S} / \mathrm{N}$ ratio for both autofluorescence emission and harmonic generation (SHG/THG). Many different features can be observed in cardiac cryosections without any labeling procedure. Remarkably, sarcomeres' myosin, as well as collagen deposits surrounding the coronary vessel, were clearly distinguishable due to their strong SHG signal from both 800 - and $1200-\mathrm{nm}$ excitation wavelengths. We evaluated the intensity profile of the SHG signal produced by the sarcomeres: it has a very good $\mathrm{S} / \mathrm{N}$ ratio [Fig. 3(d)] and, in line with the previous reports, ${ }^{11}$ appears to arise from the myosin of thick filaments. Moreover, elastin can be visualized simultaneously by TPE. The excitation wavelength of $1200 \mathrm{~nm}$ does not show some of the sample features that are clearly visible from the autofluorescence signal excited with $800 \mathrm{~nm}$, proving the dual-laser imaging approach more information-rich than the common single-wavelength TPM. We also considered the possibility of photo-thermal damages due to intrinsic fluorophore absorption. Adopting a simple model for the 3-D energy distribution around the focal plane in case of TPA and high numerical aperture, ${ }^{21}$ the temperature rise is given as

$T_{3 \mathrm{D}}=\frac{P_{\mathrm{abs}}}{\omega_{0} 4 \pi k_{T}}\left[1-\sqrt{\frac{2 \tau_{c}}{2 \tau_{c}+3 t}}\right]$,

where $P_{\mathrm{abs}}$ is the total absorbed power, $\omega_{0}$ is the beam waist, $\tau_{c}$ is the thermal time constant, and $k_{T}$ is the thermal conductivity.
Given that $k_{T} \approx 0.55 \mathrm{WK}^{-1} \mathrm{~m}^{-1}$ for most of the biological tissues, ${ }^{22} \omega_{0}(800 \mathrm{~nm}) \approx 249 \mathrm{~nm}$ and $\omega_{0}(1200 \mathrm{~nm}) \approx 374 \mathrm{~nm}$ for $1.05 \mathrm{NA}$, and the volumetric heat capacity of biological tissues ${ }^{23}$ is $\rho \approx 3.6 \mathrm{MJm}^{-3} \mathrm{~K}^{-1}, \tau_{c}$ can be calculated from

$\tau_{c}=\frac{\omega_{0}^{2} \rho}{4 k_{T}}$.

Since both $\tau_{c}(800 \mathrm{~nm})=144 \mathrm{~ns}$ and $\tau_{c}(1200 \mathrm{~nm})=$ $233 \mathrm{~ns}$ are much longer than the interpulse interval of our laser source $\left(I_{\mathrm{I}}=12 \mathrm{~ns}\right)$, the incremental temperature rise during a single pulse can be considered roughly equal to the steady-state temperature rise $T_{3 \mathrm{D}}(t=\infty)$ divided by a factor $\tau_{c} I_{\mathrm{I}}$. Therefore, the heating inside the focal volume for highrepetition-rate lasers can be treated like $\mathrm{CW}$ illumination and, since for a typical fluorophore $P_{\text {abs }} \approx 10^{-5} P_{\text {avg }}$ (average incident power) appears to be negligible at our working $2 \mathrm{PM}$ parameters.

In addition to intrinsic biological fluorophores, such as myosin, collagen, and elastin, that report solely on structural information, nicotinamide adenine dinucleotide (NADH) emits a strong TPE signal ${ }^{24}$ that is related to the metabolic state of the imaged cells. We thus applied our setup to study skeletal muscle cryosections, to discriminate glycolytic and oxidative muscle fibers depending on their NADH/mitochondria content. ${ }^{25}$ As shown in Fig. 4, two consecutive gastrocnemius muscle cryosections display fibers with higher TPE signal corresponding to those with higher SDH intensity in standard histology, consistently with the NADH origin of the TPE autofluorescence. Oxidative fibers show stronger SDH activity upon staining, corresponding to fibers having a larger number of mitochondria, more oxidative capacity, and smaller cross-sectional area. Upon repeated investigations into several cryosections [Fig. 4(d)], we confirmed the strict correlation between TPE and SDH intensity [Fig. 4(e)], thus confirming label-free TPE as a possible candidate for NADH quantification studies. ${ }^{26,27}$ Furthermore, we confirmed that the more oxidative fibers, having higher average TPE signal per encircled area, are those with the smaller crosssectional area [Fig. 4(f)]. From the same cryosection, we obtained additional information on collagen content (via SHG signal) without the need for additional staining. We performed a hematoxylin/eosin staining in the consecutive cryosection of the same muscle [Fig. 4(c)] to verify that the SHG signal really gives the same information about collagen as the conventional, widely used, staining method.

\subsection{Label-Free Analysis of Thick Ex-Vivo Samples}

We proceeded to examine freshly excised, unstained mouse lungs, as a good example of challenging thick sample. As can be seen in Fig. 5, exciting with $800 \mathrm{~nm}$ produced an SHG signal that made possible to easily recognize the collagen fibrillary structure of the lungs, and a green autofluorescence signal from the intrinsic fluorophores that highlighted the nuclei of several cells, as well as many lipid bodies scattered inside the lung tissue.

All features remained perfectly sharp and distinguishable even at relatively high zoom level, without any sign of photo-bleaching from collagen SHG, limited autofluorescence bleaching, and no photo-thermal damages, confirming the strength of a properly optimized label-free setup. Illuminating the specimen with 1200-nm light allowed us to simultaneously image collagen fibrils (SHG) and lipid bodies, which were 

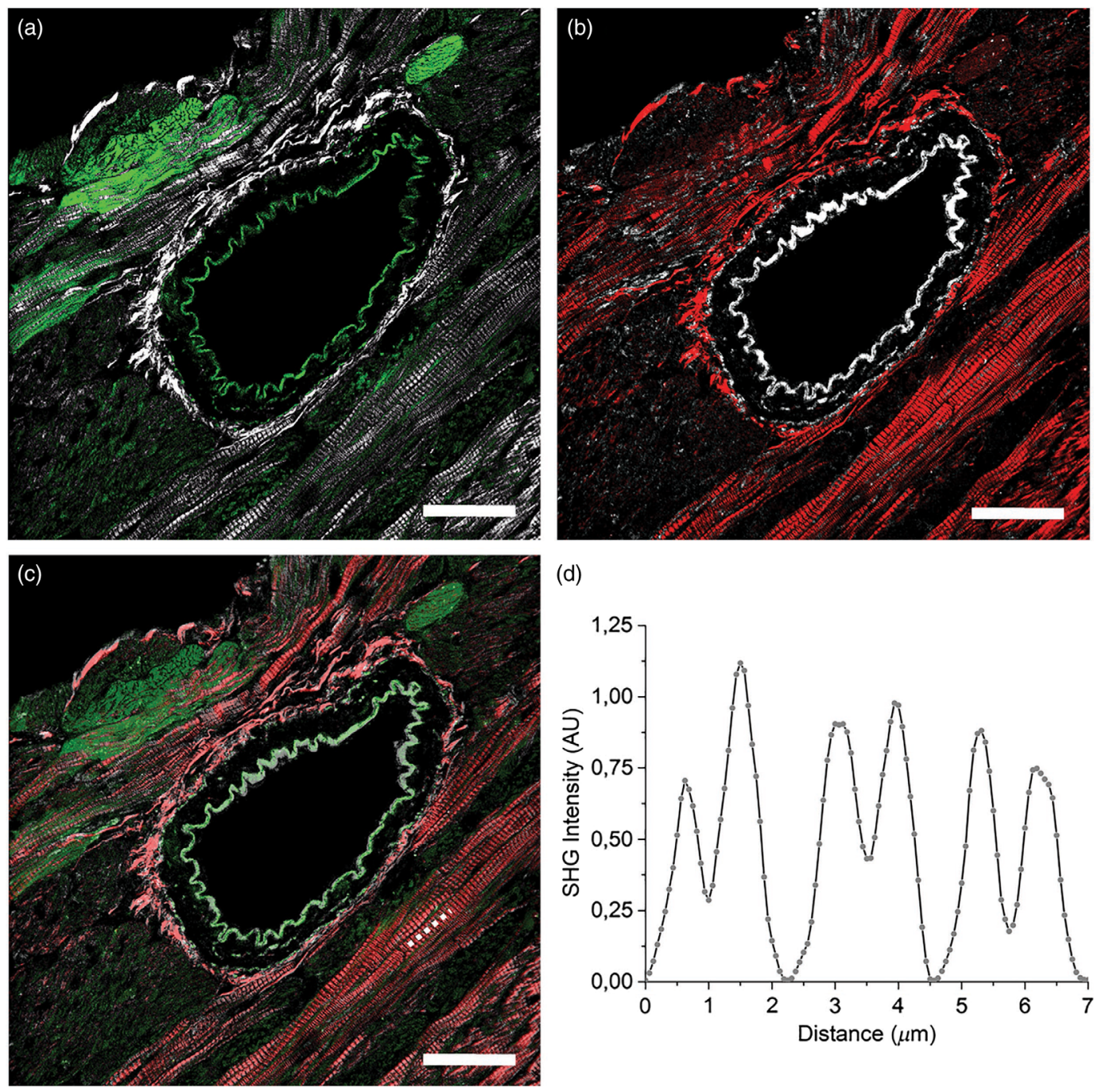

(d)

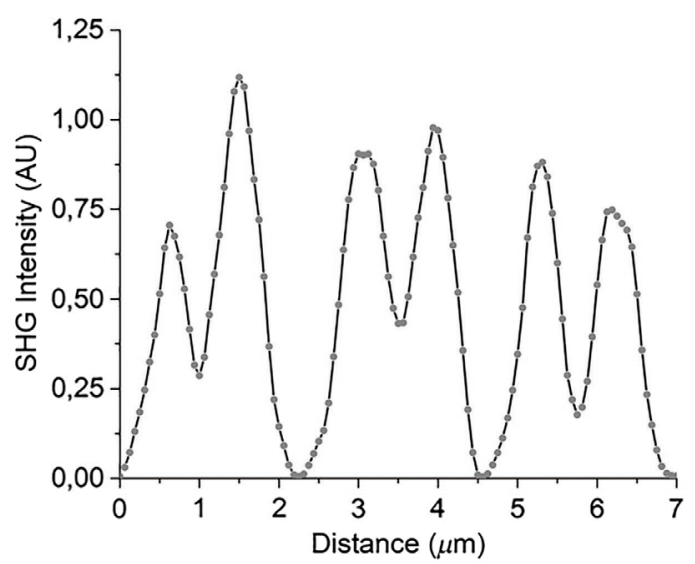

Fig. 3 Simultaneous TPE and HG images of cardiac cryosections. (a) Cardiac cryosection imaged with 800 -nm laser wavelength shows TPE autofluorescence (green, $\lambda_{\mathrm{em}} \sim 500 \mathrm{~nm}$ ) and SHG signal (white, $\lambda_{\mathrm{em}}=400 \mathrm{~nm}$ ). (b) The same field of view imaged with 1200-nm laser wavelength shows SHG (red, $\lambda_{\mathrm{em}}=600 \mathrm{~nm}$ ) and THG (white, $\lambda_{\mathrm{em}}=400 \mathrm{~nm}$ ). (c) Superposition of the same field imaged with 800 and $1200 \mathrm{~nm}$. The SHG produced by the two different laser beams, as well as the elastin autofluorescence and its THG signal, perfectly superimpose, testifying the excellent spatial alignment of the lasers. The autofluorescence excited with $800 \mathrm{~nm}$ also highlight some features that are not visible with the 1200-nm excitation wavelength, proving the dual-laser imaging approach more information-rich than the common single-wavelength TPM. (d) A portion of the SHG intensity profile of the dotted line in (c) shows the characteristic double-band of sarcomeres morphology. Scale bars $50 \mu \mathrm{m}$.

confirmed a strong source of THG signal as was shown before, ${ }^{28}$ without any detectable autofluorescence from other features, easing the study of these important organelles ${ }^{29,30}$ inside their native collagen matrix in a totally label-free environment.

\subsection{Dual-Wavelength Label-Free Imaging of Bovine Pericardium}

The main advantage of using a microscopy setup with two independent laser sources is that we can achieve the optimal illumination conditions for two distinct features without any compromise on signal strength or excitation efficiency. This is particularly useful for a label-free imaging technique, where, usually, the detected signals are significantly lower than the average fluorescence signal coming from commercial fluorophores and the requirements needed to obtain a good $\mathrm{S} / \mathrm{N}$ ratio are more stringent. To prove what our multimodal setup can achieve when applied to a biologically relevant specimen, we imaged a portion of decellularized bovine pericardium using both laser sources simultaneously (Fig. 6).

The sample was primarily made of collagen fibrils, thus one of the laser sources was entirely dedicated to the optimization of the SHG emission from the bundles. Another significant part of the sample consisted of elastin, which has its autofluorescence excitation maximum at $425 \mathrm{~nm},{ }^{31}$ thus requiring a TPE beam tuned around $800 \mathrm{~nm}$ to achieve the highest signal strength. However, collagen shows a significant absorption of blue photons, ${ }^{31}$ which competes with the TPE of the elastin autofluorescence and the detection of the SHG signal coming from the collagen itself (due to autoabsorption) resulting in a lower $\mathrm{S} / \mathrm{N}$ 

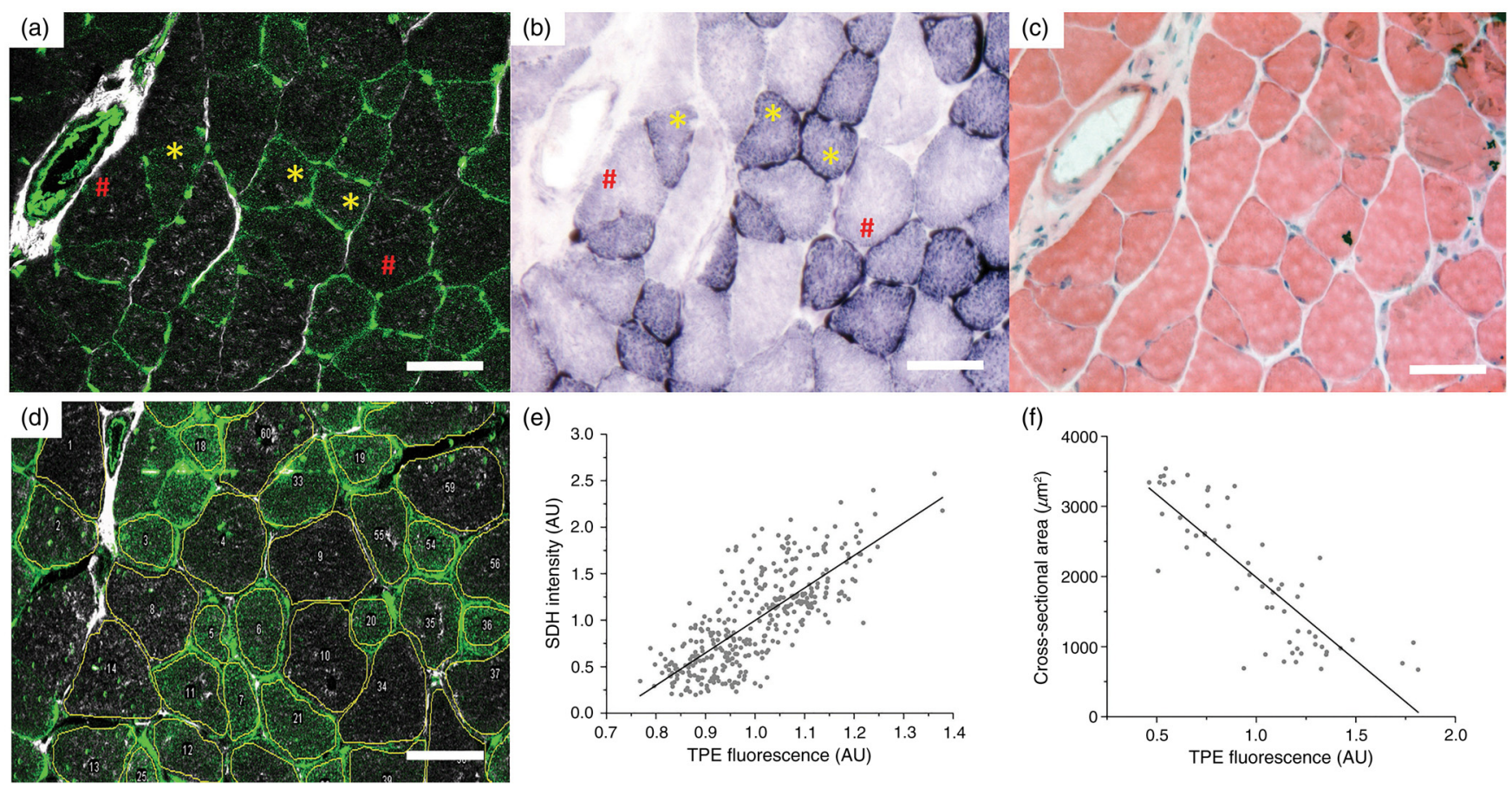

Fig. $4 \mathrm{TPE}$ of $\mathrm{NADH}$ in muscle cryosections. (a) TPE (green, $\lambda_{\mathrm{em}} \sim 500 \mathrm{~nm}$ ) and SHG (white, $\lambda_{\mathrm{em}}=400 \mathrm{~nm}$ ) signals from gastrocnemius muscles cryosection. (b) SDH staining of the consecutive cryosection from the same muscle. (c) Hematoxylin/eosin staining of the consecutive cryosection from the same muscle. (d) ROI analysis of the fibers within a muscle cryosection. (e) Correlation analysis of TPE and SDH signal intensity from the same fiber ( $N=4$ cryosections, $n=350$ fibers). Pearson correlation coefficient $P>0.75$. Yellow (*) and red (\#) highlight corresponding fibers, oxidative and glycolytic, respectively. (f) Cross-sectional area plotted against TPE fluorescence intensity highlights the higher mitochondria content of smaller fibers. Pearson correlation coefficient $P>0.85$. Scale bars $100 \mu \mathrm{m}$.
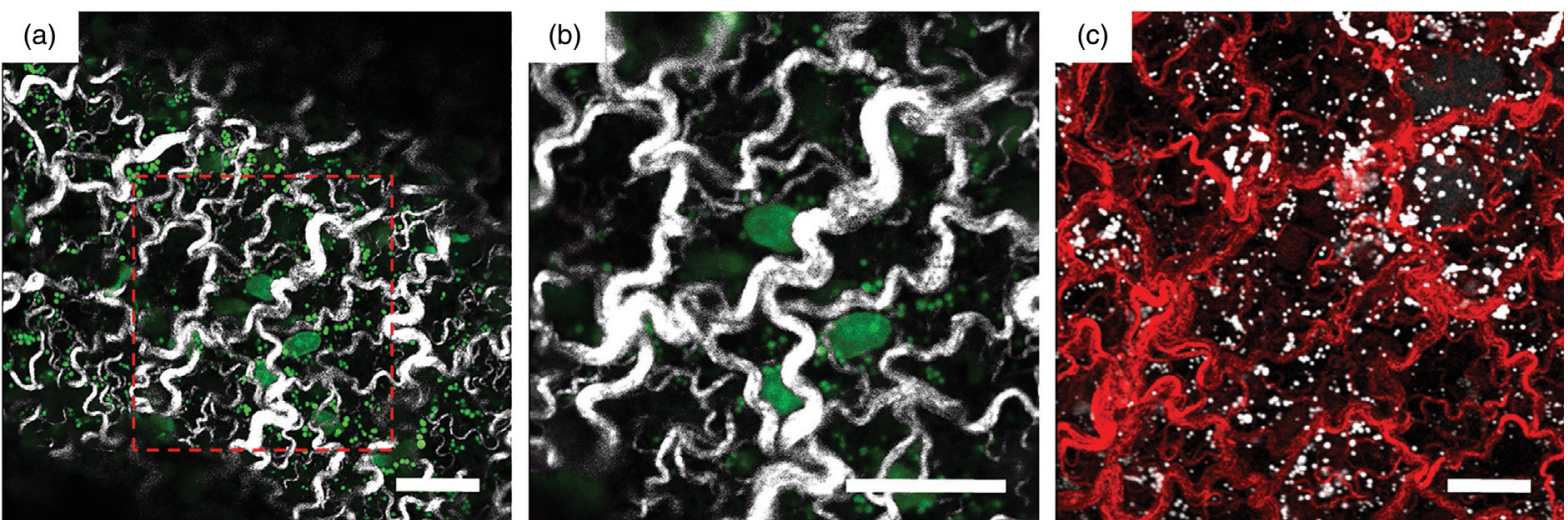

Fig. 5 TPE/SHG and SHG/THG images of ex vivo lung tissue. (a) TPE and SHG signals imaged with 800-nm wavelength. Cells nuclei and lipid bodies can be recognized by their TPE autofluorescence emission (green, $\lambda_{\mathrm{em}} \sim 500 \mathrm{~nm}$ ), whereas the coarse collagen fibers produce a strong SHG signal (white, $\lambda_{\mathrm{em}}=400 \mathrm{~nm}$ ). (b) Magnification of the area inside the red box. (c) SHG from collagen fibers and THG from lipid bodies are simultaneously excited by a 1200-nm wavelength without any autofluorescence background. The image shows a different section of the same sample as (a). Scale bars $20 \mu \mathrm{m}$.

ratio at a given excitation power and a lower SHG penetration depth. We solved this problem tuning one laser source to $800 \mathrm{~nm}$ and the other to $1200 \mathrm{~nm}$ : the first beam was optimized for the imaging of elastin autofluorescence, whereas the second beam was optimized for maximum SHG excitation efficiency. At $1200 \mathrm{~nm}$, no significant elastin excitation was measured, the SHG signal was significantly stronger (due to lack of competing processes) and the penetration depth inside the tissue was of a few hundreds of microns, thus enabling 3-D reconstruction studies on optically thick samples. $Z$-stacks performed on thick tissue showed that the SHG signal could be detected without any problem up to $200-\mu \mathrm{m}$ deep in the sample [Fig. 6(c)]. 

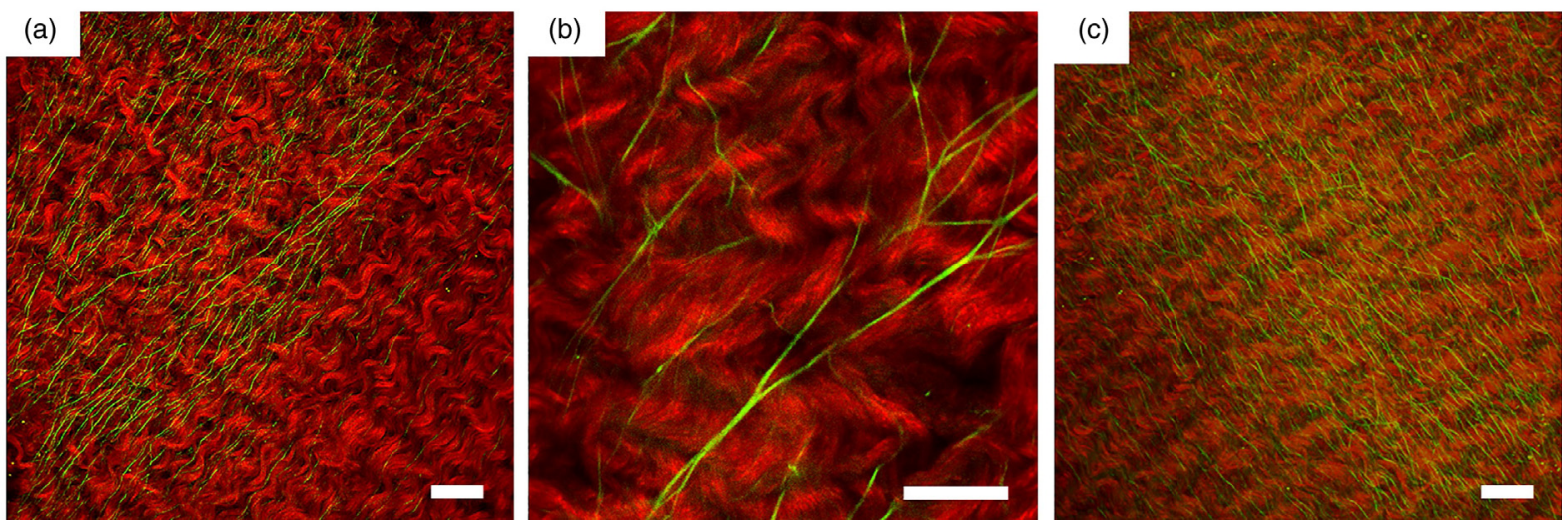

Fig. 6 Simultaneous TPE and SHG images of the decellularized pericardium. (a) SHG signal from collagen bundles imaged with 1200-nm wavelength (red, $\lambda_{\mathrm{em}}=600 \mathrm{~nm}$ ) and elastin autofluorescence excited with $800-\mathrm{nm}$ wavelength (green, $\lambda_{\mathrm{em}} \sim 500 \mathrm{~nm}$ ). (b) Details of the elastin and the collagen structure. (c) Frame from an animated movie of a $Z$-stack of $200 \mu \mathrm{m}$ of collagen (20 slices, $10 \mu \mathrm{m}$ per slice) reconstructed in Fiji (Video 1, MP4, 3.80 MB [URL: https://doi.org/10.1117/1.JBO.23.9.091403.1]). Scale bars $50 \mu \mathrm{m}$.

\section{Conclusions}

We have presented our multimodal microscope with two laser sources, capable of performing TPM and HG simultaneously by fine-tuning a specific source for one or the other. Our approach combines the best traits of both imaging techniques (e.g., intrinsic optical sectioning, reduced photo-bleaching, and label-free capabilities) while avoiding any compromise on image quality, signal strength, time investment, and with a significantly reduced need for postprocessing corrections with respect to common sequential multiwavelength approaches. ${ }^{28}$ This is particularly relevant for $\mathrm{HG}$, where the signal intensity is extremely sensitive to many external factors and the need for a careful optimization of the experimental parameters on sample basis is stringent. We showed that after a calibration of the laser's polarization states, the microscope can acquire images with a very high signal intensity and good $\mathrm{S} / \mathrm{N}$ ratio for both TPE and $\mathrm{HG}$ and enables the simultaneous label-free imaging of different structures on which several studies can be successfully performed to obtain biologically relevant information without significantly altering the samples. We also proved that all the capabilities of our setup remain unchanged while studying thick ex vivo samples, detecting significantly strong signals up to $200 \mu \mathrm{m}$ deep in the sample. We fully took advantage of the dual laser configuration while examining decellularized bovine pericardium, achieving an optical penetration depth of hundreds of microns and performing 3-D reconstructions from $z$-stacks of optically thick samples without the need of any molecular probe labeling. Using label-free techniques preserves the samples as similar as possible to their original conditions, even after several hours and many imaging sessions, maximizing the repeatability and the feasibility of multitechniques examinations. As a final remark, we want to emphasize the high upgradability of our dual-laser setup, in which more complex label-free microscopy methods, such as coherent antistokes Raman spectroscopy and stimulated Raman spectroscopy microscopy, could easily be integrated. The possibility of expanding our setup also in the direction of TPE stimulated emission depletion (2P-STED). Nanoscopy is currently being examined, as well as the use of adaptive optics to control more precisely the beams overlapping and the aberration-correction procedure. ${ }^{32}$

\section{Disclosures}

The authors declare no conflicting financial interests.

\section{Acknowledgments}

Author AF was supported by Fondazione Bruno Kessler (FBK) $\mathrm{PhD}$ fellowship. Author GB is supported by University of Padua (Bando per il Finanziamento di Assegni Dipartimentali, Bando 2016). The authors were grateful to Prof. Marco Mongillo for sharing the heart cryosection samples.

\section{References}

1. W. R. Zipfel, R. M. Williams, and W. W. Webb, "Nonlinear magic: multiphoton microscopy in the biosciences," Nat. Biotech. 21(11), 1369-1377 (2003)

2. W. Denk, J. H. Strickler, and W. W. Webb, "Two-photon laser scanning fluorescence microscopy," Science 248, 73-76 (1990).

3. M. Goeppert-Mayer, "Ueber elementarakte mit zwei Quantenspruengen," Ann. Phys. 401, 273-294 (1931).

4. V. E. White and J. G. Centoze, "Multiphoton excitation provide optical sections from deeper within scattering specimens than confocal imaging," Biophys. J. 75, 2015-2024 (1998).

5. P. Friedl et al., "Biological second and third harmonic generation microscopy," Curr. Protoc. Cell Biol. Chapter 4, Unit 4.15 (2007).

6. A. Castaldi et al., "MicroRNA-133 modulates the $\beta 1$-adrenergic receptor transduction cascade," Circ. Res. 115(2), 273-283 (2014).

7. G. Milan et al., "Regulation of autophagy and the ubiquitin-proteasome system by the FoxO transcriptional network during muscle atrophy," Nat. Commun. 6(6), 6670 (2015).

8. M. Spina et al., "Isolation of intact aortic valve scaffolds for heart-valve bioprostheses: extracellular matrix structure, prevention from calcification, and cell repopulation features," J. Biomed. Mater. Res. A 67, 1338-1350 (2003)

9. L. Iop et al., "The influence of heart valve leaflet matrix characteristics on the interaction between human mesenchymal stem cells and decellularized scaffolds," Biomaterials 30, 4104-4116 (2009).

10. B. Valeur and M. N. Berberan-Santos, Molecular Fluorescence: Principles and Applications, John Wiley \& Sons, Hoboken, New Jersey (2012).

11. S. V. Plotnikov et al., "Characterization of the Myosin-based source for second-harmonic generation from muscle sarcomeres," Biophys. J. 90(2), 693-703 (2006).

12. O. Nadiarnykh and P. J. Campagnola, "Retention of polarization signatures in SHG microscopy of scattering tissues through optical clearing," Opt. Express 17(7), 5794-5806 (2009). 
13. X. Chen et al., "Second harmonic generation microscopy for quantitative analysis of collagen fibrillar structure," Nat. Protoc. 7, 654-669 (2012).

14. M. Samim et al., "Characterization of heterogeneous media using nonlinear Stokes-Mueller polarimetry," J. Opt. Soc. Am. B 33, 2617-2625 (2016).

15. M. Samim et al., "Second harmonic generation polarization properties of myofilaments," J. Biomed. Opt. 19(5), 056005 (2014).

16. S. Galiani et al., "Strategies to maximize the performance of a STED microscope," Opt. Express 20, 7362-7374 (2012).

17. M. Leutenegger, C. Eggeling, and S. W. Hell, "Analytical description of STED microscopy performance," Opt. Express 18, 26417-26429 (2010).

18. P. F. G. Rodriguez et al., "Building a fast scanning stimulated emission depletion microscope: a step by step guide," in Current Microscopy Contributions to Advances in Science and Technology, A. MèndezVilas, Ed., pp. 791-800, Formatex Research Center, Badajoz, Spain (2012).

19. J. Tønnesen et al., "Two-color STED microscopy of living synapses using a single laser-beam pair," Biophys. J. 101(10), 2545-2552 (2011).

20. P. Mahou et al., "Metrology of multiphoton microscopes using second harmonic generation nanoprobes," Small 13, 1701442 (2017).

21. W. Denk, D. W. Piston, and W. W. Webb, "Multi-photon molecular excitation in laser-scanning microscopy," in Handbook of Biological Confocal Microscopy, Chap. 28, J. Pawley, Ed., pp. 535-549, Springer, Boston, Massachusetts (2006).

22. A. Bhattacharya and R. L. Mahajan, "Temperature dependence of thermal conductivity of biological tissues," Physiol. Meas. 24, 769-783 (2003).

23. M. Sugiyama, H. Kanayama, and I. Akiyama, "Measurement of volumetric heat capacity of biological tissues heated by ultrasound exposure," J. Acoust. Soc. Am. 140, 3372 (2016).
24. W. R. Zipfel et al., "Live tissue intrinsic emission microscopy using multiphoton-excited native fluorescence and second harmonic generation," Proc. Natl. Acad. Sci. U. S. A. 100(12), 7075-7080 (2003).

25. E. C. Rothstein et al., "Skeletal muscle NAD(P)H two-photon fluorescence microscopy in vivo: topology and optical inner filters," Biophys. J. 88(3), 2165-2176 (2005).

26. M. C. Skala et al., "In vivo multiphoton fluorescence lifetime imaging of protein-bound and free nicotinamide adenine dinucleotide in normal and precancerous epithelia," J. Biomed. Opt. 12(2), 024014 (2007).

27. S. Zhuo et al., "Label-free monitoring of colonic cancer progression using multiphoton microscopy," Biomed. Opt. Express 2(3), 615-619 (2011).

28. D. Débarre et al., "Imaging lipid bodies in cells and tissues using thirdharmonic generation microscopy," Nat. Methods 3(1), 47-53 (2006).

29. D. J. Murphy, "The biogenesis and function of lipid bodies in animals, plants and microorganisms," Prog. Lipid Res. 40, 325-438 (2001).

30. S. Martin and R. G. Parton, "Caveolin, cholesterol, and lipid bodies," Semin. Cell Dev. Biol. 16, 163-174 (2005).

31. Y. Pu et al., "Changes of collagen and nicotinamide adenine dinucleotide in human cancerous and normal prostate tissues studied using native fluorescence spectroscopy with selective excitation wavelength," J. Biomed. Opt. 15(4), 047008 (2010).

32. M. Samim et al., "Differential polarization nonlinear optical microscopy with adaptive optics controlled multiplexed beams," Int. J. Mol. Sci. 14, 18520-18534 (2013).

Biographies for the authors are not available. 